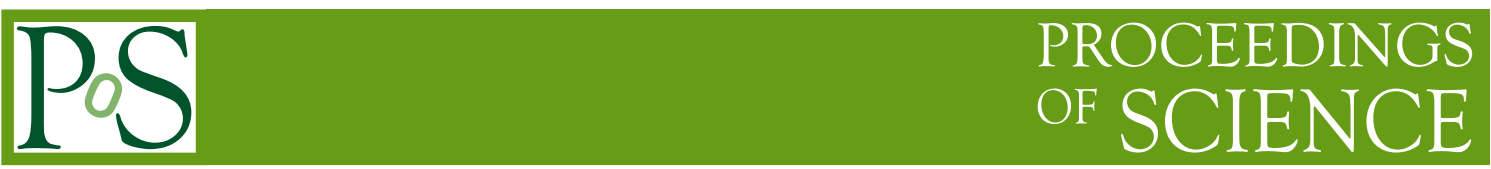

\title{
Constraining Sterile Neutrinos from Precision Higgs Data
}

\section{Arindam Das}

School of Physics, KIAS, Seoul 02455, Korea E-mail: arindamekias.re.kr

The neutrino masses and flavor mixings, which are missing in the Standard Model (SM), can be naturally incorporated in the type-I seesaw extension of the SM with heavy Majorana neutrinos being singlet under the SM gauge group. If the heavy Majorana neutrinos are around the electroweak scale and their mixings with the SM neutrinos are sizable, they can be produced at high energy colliders, leaving characteristic signatures with lepton-number violations. Employing the general parametrization for the neutrino Dirac mass matrix in the minimal seesaw scenario, we perform a parameter scan and identify allowed regions to satisfy a variety of experimental constraints from the neutrino oscillation data, the electroweak precision measurements and the lepton-flavor violating processes. We find that the resultant mixing parameters between the heavy neutrinos and the SM neutrinos are more severely constrained than those obtained from the current search for heavy Majorana neutrinos at the LHC. Such parameter regions can be explored at the High-Luminosity LHC and a $100 \mathrm{TeV}$ pp- collider in the future.

39th International Conference on High Energy Physics

4-11 July 2018

Seoul, Korea 


\section{Introduction}

With the measurements of nonzero reactor angle all neutrino oscillation data expect the Dirac $C P$-phase have been determined in [1], which indicate physics beyond the Standard Model (SM). The famous type-I seesaw extension of the SM is arguably the simplest idea to naturally incorporate the tiny neutrino masses and the flavor mixings into the SM, where heavy Majorana neutrinos which are singlet under the SM gauge group are introduced. The heavy neutrinos are integrated out at low energies, leading to a dimension five Weinberg operator among the SM lepton and the Higgs doublets at low energies. After the electroweak symmetry breaking, light Majorana masses for the SM neutrinos are generated thought the type-I seesaw mechanism. Although the heavy Majorana neutrinos are singlet under the SM gauge group, the heavy mass eigenstates after the seesaw mechanism couple with the weak bosons and the Higgs boson through the mixing with the SM neutrinos. If the heavy neutrinos are around or below the electroweak scale and the mixing with the SM neutrinos is not extremely small, the heavy Majorana neutrinos can be produced at high energy colliders. The smoking gun collider signature of heavy neutrino production at the collider experiments is the same-sign dilepton in the final state which reflects the lepton-number violation due to their Majorana masses. The heavy neutrino signature, once observed at collider experiments, can provide us with a clue to explore the origin of the neutrino masses and flavor mixings. The mixing of the heavy neutrinos with the SM neutrinos affects not only the production cross section at high energy colliders but also a variety of phenomenologies such as the neutrino oscillation data, the precision measurement of weak boson decays, and the lepton-flavor-violating decays of charged leptons, which severely constrain the mixing parameters. Therefore, in order to discuss the possibility of the heavy neutrino production at high energy colliders, it is essential to identify allowed regions for the mixing parameters from the current phenomenological constraints. In this letter, for simplicity, we consider the minimal seesaw scenario and introduce two right-handed neutrinos to the SM, which is the minimal setup to reproduce the observed neutrino oscillation data with a prediction of one massless neutrino. Employing the general parametrization for the neutrino Dirac mass matrix in the seesaw model, we perform a parameter scan to identify the allowed regions for the mixing parameters. A very brief introduction on the minimal seesaw is given in [2] where $m_{D}$ is the Dirac mass term and $M_{N}$ is the Majorana mass term. Hence we express the light neutrino flavor eigenstate $(v)$ in terms of the mass eigenstates of the light $\left(v_{m}\right)$ and heavy $\left(N_{m}\right)$ Majorana neutrinos such as $v \simeq \mathscr{N} v_{m}+\mathscr{R} N_{m}$, where $\mathscr{R}=m_{D} m_{N}^{-1}, \mathscr{N}=\left(1-\frac{1}{2} \varepsilon\right) U_{\text {MNS }}$ with $\varepsilon=\mathscr{R}^{*} \mathscr{R}^{T}$ and $U_{\mathrm{MNS}}$ is the neutrino mixing matrix which diagonalizes the light neutrino mass matrix as $U_{\mathrm{MNS}}^{T} m_{v} U_{\mathrm{MNS}}=\operatorname{diag}\left(m_{1}, m_{2}, m_{3}\right)$. In the presence of $\varepsilon$, the mixing matrix $\mathscr{N}$ is not unitary, namely $\mathscr{N}^{\dagger} \mathscr{N} \neq 1$. We can insert these forms into the charged and neutral current interactions of the SM sector with the heavy neutrinos in the mass eigenstates are given in [2]. Through the mixing $\mathscr{R}_{\alpha i}$, the heavy neutrinos can be produced at high energy colliders as studied in $[3,4,5]$ at the Large Hadron Collider (LHC) and ILC [5, 6, 7, 8].

\section{General parameters}

The elements of the matrices $\mathscr{N}$ and $\mathscr{R}$ are constrained by the experimental data. In this paper we adopt the current neutrino oscillation data from $[9,1]$. The neutrino mixing matrix is known as 
the $U_{P} M N S$ which contains the neutrino flavor mixing angles, Dirac and Majorana CP phases. The minimal seesaw scenario predicts one massless eigenstate. For the light neutrino mass spectrum, we consider both the normal hierarchy (NH) and the inverted hierarchy (IH). In the NH case, the diagonal mass matrix is given by $D_{\mathrm{NH}}=\operatorname{diag}\left(0, \sqrt{\Delta m_{12}^{2}}, \sqrt{\Delta m_{12}^{2}+\Delta m_{23}^{2}}\right)$, while in the IH case $D_{\mathrm{IH}}=\operatorname{diag}\left(\sqrt{\Delta m_{23}^{2}-\Delta m_{12}^{2}}, \sqrt{\Delta m_{23}^{2}}, 0\right)$. In order to make our discussion simple, we assume the degeneracy of the heavy neutrinos in mass such as $M_{N}=m_{N}^{1}=m_{N}^{2}$, so that the light neutrino mass matrix is simplified as $m_{v}=\frac{1}{M_{N}} m_{D} m_{D}^{T}=U_{\mathrm{MNS}}^{*} D_{\mathrm{NH} / \mathrm{IH}} U_{\mathrm{MNS}}^{\dagger}$, for the $\mathrm{NH} / \mathrm{IH}$ cases. From this formula, we can parameterize the neutrino Dirac mass matrix as [10]. We mention that our analysis is reliable at the tree level. $m_{D}=\sqrt{M_{N}} U_{\mathrm{MNS}}^{*} \sqrt{D_{\mathrm{NH} / \mathrm{IH}}} O$, from [2] and $O$ is a general $2 \times 2$ orthogonal matrix given by $O=\left(\begin{array}{cc}\cos (X+i Y) & \sin (X+i Y) \\ -\sin (X+i Y) & \cos (X+i Y)\end{array}\right)$, where $X$ and $Y$ are real parameters. Due to its non-unitarity, the elements of the mixing matrix $\mathscr{N}$ are severely constrained by the combined data from the neutrino oscillation experiments, the precision measurements of weak boson decays, and the lepton-flavor-violating decays of charged leptons. We update the results by using more recent data on the lepton-favor-violating decays: $\left|\mathscr{N}^{\dagger}\right|$ in [2] where the diagonal elements are from the precision measurements of weak boson decays (the SM prediction is 1) while the off-diagonal elements are the upper bounds from the lepton-favor-violating decays, namely, the $(1,2)$ and $(2,1)$ elements from the $\mu \rightarrow e \gamma$ process, the $(2,3)$ and $(3,2)$ elements from the $\tau \rightarrow \mu \gamma$ process, and the $(1,3)$ and $(3,1)$ elements from the $\tau \rightarrow e \gamma$ process. Since $\mathscr{N} \mathscr{N}^{\dagger} \simeq \mathbf{1}-\varepsilon$, we have the constraints on $\varepsilon$ such that $|\varepsilon|=\left(\begin{array}{ccc}0.006 \pm 0.00625 & <1.288 \times 10^{-5} & <8.76356 \times 10^{-3} \\ <1.288 \times 10^{-5} & 0.005 \pm 0.00625 & <1.046 \times 10^{-2} \\ <8.76356 \times 10^{-3} & <1.046 \times 10^{-2} & 0.005 \pm 0.00625\end{array}\right)$. The most stringent bound is given by the $(1,2)$-element which is from the constraint on the lepton-flavor-violating muon decay $\mu \rightarrow e \gamma$. Using the general parametrization of the Dirac mass matrix in Eq. (2), we have $\varepsilon(\delta, \rho, Y)=\left(\mathscr{R}^{*} \mathscr{R}^{T}\right)_{\mathrm{NH} / \mathrm{IH}}=\frac{1}{M_{N}^{2}} m_{D} m_{D}^{T}=\frac{1}{m_{N}} U_{\mathrm{MNS}} \sqrt{D_{\mathrm{NH} / \mathrm{IH}}} O^{*} O^{T} \sqrt{D_{\mathrm{NH} / \mathrm{IH}}} U_{\mathrm{MNS}}^{\dagger}$. Here, note that $\varepsilon(\delta, \rho, Y)$ is independent of $X$ since $O^{*} O^{T}$ is not a function of $X$. Now we perform a scan for the parameter set $\{\delta, \rho, Y\}$ and identify an allowed region for which $\varepsilon(\delta, \rho, Y)$ satisfies the experimental constraints in $\varepsilon$.

\section{Conclusion}

The limits on the mixing angles from EWPD [13, 12], L3 [14], Higgs-LHC[15, 16] and LHC are given in $[17,18]$ Comparing the our results with the existing limits we find that the upper limits of the mixing parameters in [2] are more constrained than the EWPD (strongest for the prompt heavy neutrinos heavier than $100 \mathrm{GeV}$.) limits for the $\mathrm{NH}$ and $\mathrm{IH}$ cases for the electron and muon flavors to produce the heavy neutrinos at the colliders.

\section{References}

[1] C. Patrignani et al. [Particle Data Group], "Review of Particle Physics," Chin. Phys. C 40, no. 10, 100001 (2016). doi:10.1088/1674-1137/40/10/100001 
[2] A. Das and N. Okada, "Bounds on heavy Majorana neutrinos in type-I seesaw and implications for collider searches," Phys. Lett. B 774, 32 (2017) doi:10.1016/j.physletb.2017.09.042 [arXiv:1702.04668 [hep-ph]].

[3] A. Das, P. Konar and S. Majhi, "Production of Heavy neutrino in next-to-leading order QCD at the LHC and beyond,” JHEP 1606, 019 (2016) doi:10.1007/JHEP06(2016)019 [arXiv:1604.00608 [hep-ph]].

[4] A. Das, "Searching for the minimal Seesaw models at the LHC and beyond," Adv. High Energy Phys. 2018, 9785318 (2018) doi:10.1155/2018/9785318 [arXiv:1803.10940 [hep-ph]].

[5] A. Das and N. Okada, "Inverse seesaw neutrino signatures at the LHC and ILC," Phys. Rev. D 88, 113001 (2013) doi:10.1103/PhysRevD.88.113001 [arXiv:1207.3734 [hep-ph]].

[6] S. Antusch and O. Fischer, "Testing sterile neutrino extensions of the Standard Model at future lepton colliders," JHEP 1505, 053 (2015) doi:10.1007/JHEP05(2015)053 [arXiv:1502.05915 [hep-ph]].

[7] S. Banerjee, P. S. B. Dev, A. Ibarra, T. Mandal and M. Mitra, "Prospects of Heavy Neutrino Searches at Future Lepton Colliders,” Phys. Rev. D 92, 075002 (2015) doi:10.1103/PhysRevD.92.075002 [arXiv:1503.05491 [hep-ph]].

[8] S. Chakraborty, M. Mitra and S. Shil, "Fat Jet Signature of a Heavy Neutrino at Lepton Collider," arXiv:1810.08970 [hep-ph].

[9] F. P. An et al. [DAYA-BAY Collaboration], Phys. Rev. Lett. 108, 171803 (2012).

[10] J. A. Casas and A. Ibarra, “Oscillating neutrinos and muon $\rightarrow$ e, gamma,” Nucl. Phys. B 618, 171 (2001) doi:10.1016/S0550-3213(01)00475-8 [hep-ph/0103065].

[11] J. Lopez-Pavon, E. Molinaro and S. T. Petcov, "Radiative Corrections to Light Neutrino Masses in Low Scale Type I Seesaw Scenarios and Neutrinoless Double Beta Decay,” JHEP 1511, 030 (2015) doi:10.1007/JHEP11(2015)030 [arXiv:1506.05296 [hep-ph]].

[12] F. del Aguila, J. de Blas and M. Perez-Victoria, "Effects of new leptons in Electroweak Precision Data,” Phys. Rev. D 78, 013010 (2008) doi:10.1103/PhysRevD.78.013010 [arXiv:0803.4008 [hep-ph]].

[13] J. de Blas, "Electroweak limits on physics beyond the Standard Model," EPJ Web Conf. 60, 19008 (2013) doi:10.1051/epjconf/20136019008 [arXiv:1307.6173 [hep-ph]].

[14] P. Achard et al. [L3 Collaboration], "Search for heavy isosinglet neutrino in $e^{+} e^{-}$annihilation at LEP,” Phys. Lett. B 517, 67 (2001) doi:10.1016/S0370-2693(01)00993-5 [hep-ex/0107014].

[15] P. S. Bhupal Dev, R. Franceschini and R. N. Mohapatra, "Bounds on TeV Seesaw Models from LHC Higgs Data,” Phys. Rev. D 86, 093010 (2012) doi:10.1103/PhysRevD.86.093010 [arXiv:1207.2756 [hep-ph]].

[16] A. Das, P. S. B. Dev and C. S. Kim, "Constraining Sterile Neutrinos from Precision Higgs Data," Phys. Rev. D 95, no. 11, 115013 (2017) doi:10.1103/PhysRevD.95.115013 [arXiv:1704.00880 [hep-ph]].

[17] G. Aad et al. [ATLAS Collaboration], "Search for heavy Majorana neutrinos with the ATLAS detector in pp collisions at $\sqrt{s}=8 \mathrm{TeV}$," JHEP 1507, 162 (2015) doi:10.1007/JHEP07(2015)162 [arXiv:1506.06020 [hep-ex]].

[18] V. Khachatryan et. al. [CMS Collaboration], "Search for heavy Majorana neutrinos in $\mathrm{e}^{ \pm} \mathrm{e}^{ \pm}+$jets and $\mathrm{e}^{ \pm} \mu^{ \pm}+$jets events in proton-proton collisions at $\sqrt{s}=8 \mathrm{TeV}$, JHEP 1604, 169 (2016) doi: 10.1007/JHEP04 (2016)169 [arxiv:1603.02248 [hep-ex]]. 